\title{
New Leadership - Führen in agilen Unternehmen
}

\author{
Heidi Möller • Thomas Giernalczyk
}

Eingegangen: 14. September 2021 / Angenommen: 20. Oktober 2021 / Online publiziert: 16. Dezember 2021

(C) Der/die Autor(en) 2021

Zusammenfassung Das Prinzip der Agilität stellt Führungskräfte vor die Herausforderung, Führung neu zu denken und umzusetzen. Sie verlieren Positionsmacht und müssen sich auf Augenhöhe einlassen und ihre Macht teilen. Unsere Reflexionsfragen helfen Führungskräften, vom „Nowland“ zum „Nextland“ zu kommen. Wir zeigen auf, wie sie durch agile Führung Handlungsspielraum und Einfluss auf gesellschaftliche Entwicklungen gewinnen, und stellen das Konzept „Psychologische Sicherheit" als hilfreiche Methode zur Selbststeuerung vor.

Schlüsselwörter Agile Führung · Macht · Change-Prozess · Psychologische Sicherheit · Selbststeuerung

\section{New leadership in agile organizations}

Abstract The principle of agility challenges managers to rethink and implement leadership. They lose positional power and have to engage at eye level and share power. Our reflection questions help leaders move from "nowland" to "nextland". We show how they can gain room to maneuver and influence social developments through agile leadership and introduce the concept of "Psychological Safety" as a helpful method for self-direction.

Keywords Agile leadership · Power · Change-process · Psychological safety · Self-management

Prof. Dr. Heidi Möller ( $\varangle)$

Institut für Psychologie, Universität Kassel, Holländische Straße 36-38, 34127 Kassel, Deutschland E-Mail: heidi.moeller@uni-kassel.de

Prof. Dr. Thomas Giernalczyk

M19 - Manufaktur für Organisationsberatung GmbH, Bauerstraße 19, 80796 München, Deutschland E-Mail: thomas.giernalczyk@m19-organisationsberatung.de 


\section{Agilität als modernes Organisationsprinzip}

Agilität gilt als modernes Organisationsprinzip. Im Kern hat es den Anspruch, Unternehmen in komplexen und sich schnell ändernden Märkten adaptiv zu steuern, sodass sie sich auch in der VUCA-Welt erfolgreich entwickeln können. Eng verwandt und inzwischen überschneidend mit Agilität verwendet ist der Ansatz des „New Work“, den Bergmann (2017 [2004]) als Kritik an traditioneller Arbeit entwickelt hat. Beide Konzepte eint, dass sie die Entwicklung der Mitarbeitenden (Empowerment), das Selbstmanagement von Teams und die Sinnorientierung (Purpose) für zentral erachten. Das Agilitätsparadigma betont zudem die enge Zusammenarbeit mit Kund/ innen bereits während der Entwicklung von Produkten und hält iteratives Vorgehen für den überlegenen Weg gegenüber langfristig angelegten wasserfallartigen Entwicklungsprozessen.

Das ,agile Manifest“ (Beck et al. 2001) als ein wichtiger Meilenstein in der Software- und Organisationsentwicklung stellt paradigmatisch die Kernüberzeugungen zusammen: Wir schätzen ,Individuen und Interaktionen mehr als Prozesse und Werkzeuge. Funktionierende Software mehr als umfassende Dokumentation. Zusammenarbeit mit dem Kunden mehr als Vertragsverhandlung. Reagieren auf Veränderung mehr als das Befolgen eines Plans“.

Agilität ist längst kein einheitliches Konzept, sondern eine „Diskurswolke“, die sich ständig weiterentwickelt und neu abgrenzt. Agilität verändert die primäre Aufgabe der Führungskräfte. Sie verlieren hierarchische Positionsmacht und müssen sich auf Mitarbeitende und Teams ,,auf Augenhöhe“ einlassen und mit Sinnorientierung führen. Dabei darf nicht vergessen werden, dass eine Vielzahl von Unternehmen aus einer hierarchischen „Beharrungskultur“ kommen und Schritt für Schritt eine agile „Entwicklungskultur“ etablieren (Giernalczyk und Möller 2018). Agil führen heißt für Führungskräfte, eigenes Vorgehen und Haltungen zu verändern sowie die Motivstruktur zu reflektieren.

\section{Was macht das Agilitätsparadigma mit den (traditionellen) Führungskräften?}

Wie sieht das Motivationsprofil eines traditionellen Managers auf verschiedenen Management-Levels aus? Er verfügt über ein durchschnittlich ausgeprägtes Leistungsmotiv, denn nicht der beste Operateur in der Klinik ist der beste Krankenhausmanager und nicht die beste Autoverkäuferin die beste Niederlassungsleiterin. Das Freundschaftsmotiv ist da, aber nicht sehr ausgeprägt. Zur Empathie muss die Fähigkeit zur Affiliation vorhanden sein, aber wer stets liebgehabt werden will, sollte von Führungsfunktionen absehen. Das Machtmotiv jedoch muss sehr ausgeprägt sein (Abb. 1). 


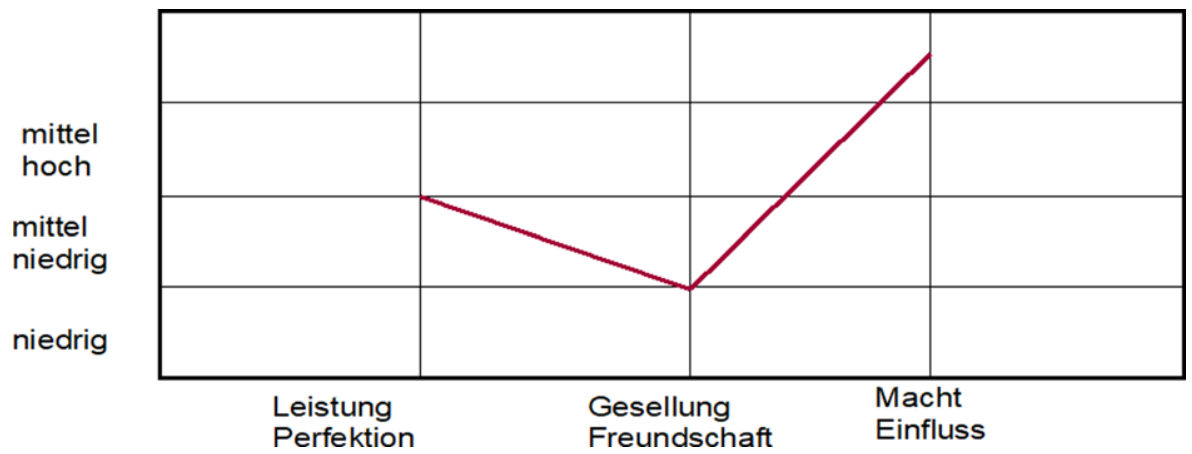

Abb. 1 Das Motivationsprofil von Managern. (Eigene Darstellung, angelehnt an Krug und Kuhl 2006, S. 109)

\section{Welche Form der Machtausübung braucht eine agile Organisation?}

Krug und Kuhl (2006, S. 40 ff.) haben eine zielführende Typologie der Machtausübung vorgelegt. Sie unterscheiden:

- Stadium der anlehnenden Macht (M1)

- Stadium der selbstbezogenen Machtausübung (M2)

- Stadium eigennütziger/personifizierter Machtausübung (M3)

- Stadium der gemeinschaftsdienlichen Machtausübung (M4)

Unsere Antwort lautet: Es braucht die M4-Typen für die agile Transformation. Dies sind Führungskräfte, die über kaum persönliche Machtziele verfügen. Sie möchten höheren Zielen und Werten zum Durchbruch verhelfen. Sie sind in der Lage, Eigeninteressen zugunsten von Organisationsinteressen zurückstellen. Sie halten sich selbst an entwickelte Regeln und übertragen Verantwortung. Durch ihr Charisma gelingt es ihnen, die Mitarbeitenden auf die Organisation und nicht auf die eigene Person einzuschwören. M4-Typen sind stark zukunftsorientiert und sehen sich im zeitlichen Kontinuum von Kommen und Gehen, soll heißen, sie bauen frühzeitig Nachfolger/innen auf.

Auch Steinmann et al. (2020) ist es zu verdanken, dass die Forschung zu impliziten Motiven im Hinblick auf effektives Führen in der akademischen Psychologie wieder hoffähig geworden ist. Der TAT (Thematischer Apperzeptionstest) wurde von Murray und Morgan 1935 entwickelt (Murray 1943), von McClelland (Picture-Story-Exercise PSE; McClelland 1975) und von Heckhausen ab 1963 weiterentwickelt (Heckhausen und Heckhausen 2006) und stellte jahrzehntelang das Diagnoseinstrument dar, um implizite Motive zu erfassen. Nachdem projektive Verfahren mitsamt der psychodynamischen Perspektive zunehmend verunglimpft wurden, verschwand auch die Bedeutung des TAT in der psychologischen Forschung und Praxis, z. B. der Personalauswahl. Der TAT legt 10 Bildtafeln zugrunde und fordert die Proband/innen auf, entlang folgender Gedanken eine Geschichte zu entwickeln und aufzuschreiben:

- Was führte zu der gezeigten Situation?

- Was geschieht gerade? 
- Was fühlen und denken die Personen?

- Wie ist der Ausgang der Geschichte?

Auf das aufwendige Auswertungsverfahren wird an dieser Stelle nicht eingegangen. Wichtig ist, dass durch diesen projektiven Test ein Zugang zum Vorbewussten möglich ist. Mit dem Ersatz des TAT durch Motivationsfragebögen entging der Motivationspsychologie eine wesentliche Erkenntnisdimension. Werden doch motivationale Muster (affektive Präferenzen) sehr früh gelernt und sind deswegen kaum sprachlich repräsentiert. So messen auf Selbstauskunft beruhende Motivationsfragebögen, die den TAT ablösten, eher das Selbstkonzept zu Motivation der Befragten als denn die Motivation selbst, verstanden als den Wunsch, sich immer wieder mit bestimmten Formen von Anreizen auseinanderzusetzen. Implizite Motivation unterliegt eben keiner Kontrolle und ist zunächst auch der Selbstreflexion nur schwer zugänglich. Auch etablierte Motivationsforscher wie Rheinberg und Engeser (2010) sahen den Preis, den der Verzicht auf projektive Verfahren forderte. Heute ist das implizite Motiv wieder im Zentrum wissenschaftlicher Aufmerksamkeit und unterschiedlichste Forscher/innen widmen sich ihm durch verschiedene Zugänge.

So zeigen Steinmann et al. (2020), dass transformationale Führung eben sehr wohl einer hohen Ausprägung des Freundschafts/Gesellungsmotivs (die schwierige Übersetzung von affiliation) bedarf. Hieß es früher: „Bitte nicht so viel affiliation, denn wer liebgehabt werden muss, sollte keine Führungskraft werden", so zeigen die aktuelle Forschung und die Anforderungen agiler Führung eine ganz andere Perspektive. Das Gesellungsmotiv führt zur Ausprägung hoher interpersonaler Kompetenzen. Das bedeutet, dass die Führungskraft viel in die Konfliktprophylaxe zu investieren in der Lage ist, z. B. dadurch, dass sie eine gute Wahrnehmung für die Bedürfnisse und Emotionen der Mitarbeitenden hat, deren Fähigkeiten und Fertigkeiten kennt und diese in die Entscheidungsfindung mit einbezieht. Teaminteraktion und organisationales Netzwerken braucht die Freude am Kontakt mit Menschen. Erfolgreiche agile Führung ist stets am Wohlergehen des Teams orientiert. Das Gesellungsmotiv verhilft dazu, die Arbeitsanforderungen mit dem Führungshandeln zu synchronisieren. Die Sorge, ob ich gemocht werde, kann auch dazu führen, dass mein Führungshandeln im guten Sinne situativ angepasst wird und flexibel ist. Um warme und unterstützende Beziehungen zum und im Team zu stiften, braucht es neben der intellektuellen Stimulanz Affiliation, um agile Teams zum Erfolg zu bringen.

Steinmann et al. (2020) sprechen auch von der Notwendigkeit, personalisierte Macht zu hemmen. Sozialisierte Macht hingegen unterstützt die fachliche und persönliche Entwicklung der Mitarbeitenden. Das empowernde Führungshandeln stärkt die Identifikation mit der Organisation und ihren Zielen. Durch ihre Autorität und Werte- und Gerechtigkeitsorientierung haben gesellungsstarke Führungskräfte eine hohe Anziehungskraft für Mitarbeitende.

\section{Orientierungsrahmen für agile Führungskräfte}

Welche Führungsmodelle stellen eine geeignete Orientierung für Führungskräfte dar? Je nach Entwicklungsstand der Organisation kann sich diese Frage nur auf 
die Führungskräfte beziehen, die direkt agile Teams leiten, oder auch das Topmanagement einbeziehen, wenn die gesamte Organisation agil strukturiert ist. Um entsprechend der Zielsetzung von agilen Organisationen ihre Mitglieder in Richtung Selbststeuerung weiterentwickeln zu können, bekommt Empowerment-Führung zentrale Bedeutung. Sie soll gewährleisten, dass Führungskräfte entsprechende Lernprozesse bei ihren Geführten anstoßen und die Mitarbeitenden darin unterstützen, zunehmend selbstständiger und selbststeuernder zu agieren. Ein anderer prominenter Ansatz ist die geteilte Führung. Bei diesem Konzept steht die Überlegung im Mittelpunkt, bestehende Führungsprozesse aufzuteilen und auf verschiedene Schultern zu legen. Nicht eine Führungskraft steuert das Team, sondern verschiedene Teammitglieder übernehmen Teil-Führungsaufgaben und tragen somit ebenfalls zur Steuerung bei. Gerade bei Organisationen, in denen Projektarbeit und Netzwerkstrukturen wichtig sind, spielt laterale Führung eine große Rolle. Wie gelingt es, Führung auszuüben, obwohl keine Hierarchiebeziehung besteht und Zusammenarbeit in der Matrix auf effiziente Weise organisiert werden muss? Laterale Führung betont echte Zusammenarbeit über Abteilungs- und Teamgrenzen hinweg, die auf gemeinsame Ziele einzahlt. Da in agilen Organisationen strukturierende Stützwerke zunehmend zurücktreten, gewinnt die Perspektive psychodynamischer Führung zunehmend an Bedeutung. Psychodynamische Führung räumt Emotionen einen hohen Stellenwert ein und fordert von den Führungskräften, Spannungen und Sorgen der Mitarbeitenden aktiv aufzunehmen und zu containen. Darüber hinaus wird die Schaffung von Reflexionsräumen betont, in denen das ,wie“ der Zusammenarbeit und die dabei entstehenden Stimmungen und Befindlichkeiten ernst genommen werden, um auf Dauer psychologische Sicherheit und Identifizierung mit der jeweiligen Einheit zu erzeugen.

Als eine Art übergreifende Dachperspektive wird das Rollenkonzept für agile Führungskräfte beschrieben. Das Rollenkonzept beschreibt das Aushandeln gegenseitiger Erwartungen, die Verteilung der Aufgaben und Verantwortlichkeiten. Es ist eine Basis, mit der die Konsequenzen organisationaler Veränderungen bis auf die Handlungsebene der Führungskräfte hinunter klar beschreibbar sind.

Musati (2020) geht den neuen Führungsanforderungen in einer qualitativen Interviewstudie nach. Sie kommt zu dem Ergebnis, dass Führungskräfte in agil werdenden Unternehmen enorm divergierenden Rollenerwartungen ausgesetzt sind: Das Management konfrontiert sie mit harten Ergebniserwartungen, und die Mitarbeitenden wünschen sich vor allem Eigenverantwortung und Gestaltungsspielräume. In ihrer Diskussion schlägt die Autorin einen Orientierungsrahmen (Agile Five Führungsset für agiler werdende Organisationen; ebd., S. 83) vor, in dem Führungskräfte auf der Basis des Rollenkonzepts regelmäßig Rollenerwartungen wichtiger Stakeholder reflektieren.

Ausgehend von Konzeptkernen der Agilität nehmen wir in Übereinstimmung mit Musati (2020, 2022) eine Auswahl von Führungsmodellen vor, die wir für nützlich halten (Abb. 2):

- Laterale Führung: Welche (teil-)autonomen Teams und Netzwerke müssen kommunikativ eingebunden werden?

- Geteilte Führung: Welche Führungsaufgaben können auf welche Mitarbeitenden verteilt werden? 


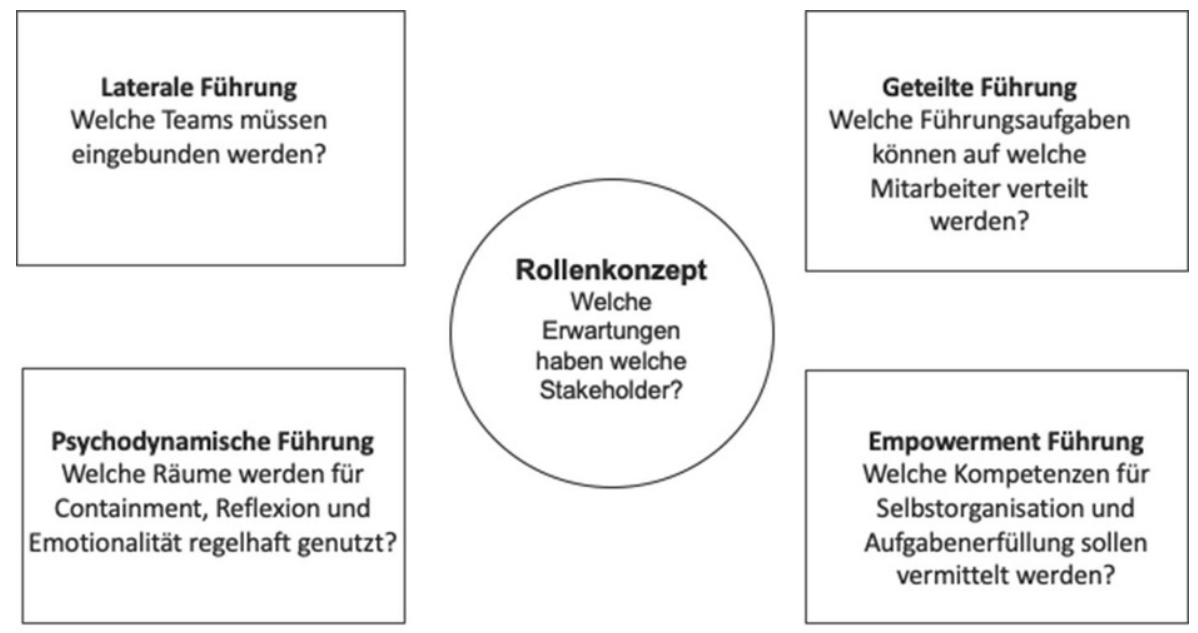

Agile Five - Führungsset für agiler werdende Organisationen, modifiziert nach Musati 2020

Abb. 2 Agile Five - Führungsset für agiler werdende Organisationen. (Modifiziert nach Musati 2020)

- Psychodynamische Führung: Welche Räume werden für Containment, Reflexion und Emotionalität geschaffen und regelhaft genutzt?

- Empowerment Führung: Welche Kompetenzen für Selbstorganisation und Aufgabenerfüllung sollen vermittelt werden?

\section{5 Übergangsmanagement}

Auf der Schwelle zwischen traditioneller Unternehmensführung und der Neugestaltung nach Agilitätsprinzipien ist vor allem eines gefragt: die Qualität des Übergangs zu managen. Das Alte trägt nicht mehr und das Neue ist noch nicht da (Abb. 3). Dieser Schwebezustand erfordert eine Menge Spannungstoleranz. Wie in jedem Veränderungsprozess ist die Zukunft ungewiss. Noch ist der Zielzustand nur zu erahnen. Klar aber ist, dass es nicht wie gewohnt weitergeht. Führungskräfte erleben wie ihre Mitarbeitenden den emotionalen Raum zwischen „Nicht-mehr“ und „Noch-nicht“. Der Schwebezustand wirft vor allem eine Frage auf: „Was wird meine Rolle sein?“.

Häufig wird in der Euphorie auf dem Weg zum agilen Unternehmen vergessen, das Vergangene zu würdigen. Denn eine Entscheidung, von nun an agil arbeiten zu wollen, verursacht indirekt eine Kränkung. War das denn alles schlecht, was wir bislang gemacht haben? Um die Mitarbeitenden für diesen Prozess zu gewinnen, braucht es die Kraft der Rituale, in denen das Alte gewürdigt wird. Um den Übergang zu gestalten, sind folgende Reflexionsfragen für alle Beteiligten sinnvoll:

- Was verliere ich?

- Welchen Zweck verbindet meine Organisation mit der Veränderung?

- Welches „Problem“ soll damit gelöst werden? 
Abb. 3 Übergangsmanagement. (Eigene Darstellung, Giernalczyk und Möller 2018, S. 87)

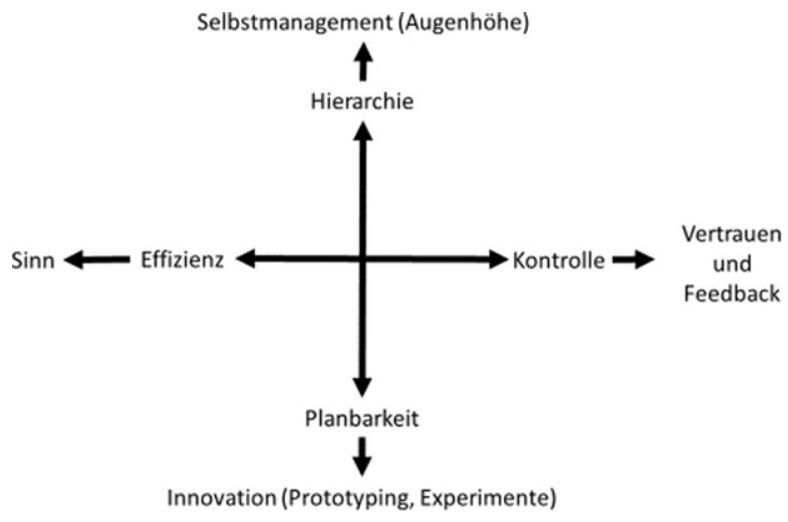

- Was würde passieren, wenn wir den Übergang nicht vollziehen?

- Welchen Sinn ziehe ich persönlich aus der Situation?

- Somatische Marker, Signale des emotionalen Erfahrungsgedächtnisses (Körperempfindungen),

- Ressourcenjagd: Was brauche ich für den Übergang?

\section{Es gibt keine Veränderungen ohne Widerstand}

Das Ausbleiben von Widerständen bei einem so grundlegenden Transformationsprozess hin zu einer agilen Organisation ist eher Anlass zur Beunruhigung, als es dessen Auftreten ist. Keinerlei Widerstandsphänomene im Zuge dieser Veränderung kann eher als ein Zeichen dafür gewertet werden, dass niemand der Beteiligten an die Realisierung glaubt. Widerstand enthält aber immer eine „verschlüsselte Botschaft" (Becker und Langosch 1995), die es zu entschlüsseln gilt. Widerstände sind Ausdruck von Bedenken, Befürchtungen oder Ängsten, die im emotionalen Bereich liegen. Als aktiv innovierende Akteurin kann ich mich sehr leicht anschlussfähig machen. Sehe ich mich jedoch eher als „Opfer“ eines Transformationsprozesses, ist es naheliegend, dass ich emotional weniger erfreut auf die Herausforderungen antworte. Jedes Organisationsmitglied reagiert vorbewusst auf die Ankündigung einer Veränderung mit einer kognitiv/emotionalen Bewertung. Dieser Prozess dauert Sekunden: „Welchen Anreiz gibt es, mich den neuen Herausforderungen zu stellen?“ Wenn nach meiner Einschätzung Folgendes zu erwarten ist:

- eine interessantere Arbeit,

- ein höherer Verdienst,

- dass ich den Anforderungen gewachsen bin - im Sinne der Selbstkontrollüberzeugung,

- oder dass negative Aspekte meiner Tätigkeit wegfallen, es leichter, effizienter, rascher wird, weniger Reibungsverluste entstehen ... 
werde ich mich anders verhalten können, als wenn zu erwarten ist:

- diese Herausforderung ist nicht zu bewältigen,

- ich muss viel Neues lernen,

- die Veränderung hat für mich im Wesentlichen negative Konsequenzen,

- mir wird Beweglichkeit aufgezwungen,

- Sanktionen und Statusverlust sind zu befürchten - bis hin zum Arbeitsplatzverlust ...

... und dann wird wohl niemand mitmachen wollen.

Erst die Bewertung der Veränderungsanforderung bestimmt darüber, ob die Belastung für eine Person eine positive oder eine negative Auswirkung hat. Nach dem Motto Senecas: „Es sind nicht die Dinge, die uns beunruhigen, sondern unsere Meinung über die Dinge“, kommt der agilen Führungskraft die Aufgabe zu, Sinn zu stiften für den Transformationsprozess. Es gilt, die Vorteile zu übersetzen und Attraktoren zu kreieren, die Lust auf Veränderung machen.

Die Nichtbeachtung von Phänomenen des Widerstands führt zu Blockaden. Eine Verstärkung des Drucks führt nur zu verstärktem Gegendruck. Also muss der Weg sein, mit dem Widerstand zu gehen und nicht gegen ihn zu arbeiten. Der unterschwellige Unmut, die destruktive Emotionalität muss aufgenommen, containt werden, damit sie sinnvoll kanalisiert werden kann.

\section{Was gewinnen Führungskräfte unter dem Agilitätsparadigma?}

Es hilft, sich zunächst selbst als Führungskraft die Vorteile agilen Managements zu übersetzen. Analoges ist dann im zweiten Schritt mit den Mitarbeitenden herauszufinden. Führungskräfte, die das Wagnis agilen Managements eingehen, entwickeln eine neue Identität, die Führungsperson kann facettenreicher werden. Führung wird im agilen Paradigma als Dienstleistung betrachtet. Führung heißt, ein Containment zur Entfaltung der besten Kräfte der Mitarbeitenden zu schaffen. Führung wird als spezifische, rollengestützte Funktion gesehen, die Kooperationsbeziehungen ermöglicht. Das Ziel der Führung ist das Entfalten von Selbstorganisation und Selbststeuerung der Subsysteme. Im Sinne des postheroischen Managements (Baecker 1994) wird ein ,neuartiger Spürsinn für die sachlichen und sozialen Dimensionen der Organisation von Arbeit und Verteilung von Verantwortung“ entwickelt. Reflexiv gewordene Moderne braucht ein reflexives Führungsverständnis, um auf die erhöhten Differenzierungsgrade und Komplexitäten der Organisationen antworten zu können. Die innovative Ausgestaltung der Führungsrolle setzt ein hohes Maß an Awareness voraus, ein hohes Reflexionsniveau bezogen auf eigene Stärken und Schwächen. Führung im agilen Kontext ist ein stetiger, lebenslanger Lernprozess, der einen selbstreflexiven Umgang von Vorgesetzten mit seinen/ihren Führungsfähigkeiten und Führungserfahrungen benötigt. Zentrale Bedeutung kommt dabei der Verarbeitung von Interaktionserfahrung zu, die durch eine lebendige FeedbackKultur im Unternehmen sowie durch systematisierte Rückkoppelungsprozesse unterstützt werden kann. „In kleinen, autonomen Teams, die in permanenter Abstimmung mit Kunden oder Lieferanten auf die zunehmende Geschwindigkeit der globalen Ver- 
änderungen reagieren können, steigt das Erleben von Bedeutsamkeit, Kompetenz, Selbstbestimmung und Einfluss für die Beschäftigten“" (Schermuly 2019).

Führungskräfte stehen zudem aufgrund ihres Kompetenzprofils in einer erhöhten Verantwortung der Gesellschaft gegenüber. Für Nachhaltigkeit im Sinne der ESG Kriterien als zentrale Orientierung braucht es eine verstärkte Auseinandersetzung mit normativen Fragen wirtschaftlichen Handelns. Kreativität und Mut sind gefragt, wenn es darum geht, Wertschöpfung und wertegeleitetes Handeln nicht mehr als Gegensätze zu definieren.

Die Umstellung auf agiles Management ermöglicht Führungskräften, ganz neue Seiten von sich kennenzulernen. An die Stelle der individuellen Karriereorientierung wird die Relevanz der Führungskraft für ihr Team bedeutsam. Sie müssen sich zeigen und sich nicht hinter ihrer Rolle verstecken. Es entsteht auf diese Weise eine neue Beziehung zum Team. Soziale Anerkennung wird wichtiger als der persönliche Erfolg. Als Erleichterung kann erlebt werden, dass Führungskräfte im agilen Management weniger als Helden aufgeladen werden müssen. Führung wird geteilt, Führungskräfte dürfen sich als Lernende verstehen, wieder Anfänger/innen sein. Die Umstellung auf agiles Arbeiten ist Befreiung und Erleichterung. Aufgrund des iterativen Vorgehens, bei dem Versuche auch verworfen werden können, dürfen agile Führungskräfte unperfekt sein (fail early), Fehler machen und gewinnen kreative Freiheit.

Agil zu arbeiten, bedeutet aus Sicht der Führungskräfte, Verantwortung zu delegieren. Um hochflexibel zu sein und dezentral arbeiten zu können, wird unter dem Agilitätsparadigma vor allem auf die Selbststeuerungskompetenz der Mitarbeitenden gesetzt. Selbststeuerungskompetenz ist jedoch ein nicht sonderlich trennscharfer Begriff. Das Konzept der Selbstführung nach Neck und Manz (2013) meint das Potenzial von Mitarbeitenden, sich selbst zu beeinflussen, um Ziele zu erreichen. Unterschiedliche Strategien zahlen auf dieses Ziel ein: Zu nennen sind die Selbstbeobachtung, die Selbstreflexion, die Fähigkeit, sich selbst eine Struktur zu geben, das Selbstmanagement, die Selbstdisziplin, die Selbstmotivation, die Selbstbelohnung versus -bestrafung, u.v.m.

Kompetente Selbstführung umfasst konstruktives Denken und planvolles Handeln. Sie fördert die Lösung von Problemen und hilft, Hindernisse zu überwinden und Schwierigkeiten zu bewältigen. Die Messung der Selbstführungskompetenz erfolgt anhand von fünf Items des Fragebogens zur Diagnose individueller Selbstführungskompetenz (FDSK; vgl. Müller 2013). Der Fragebogen erfasst, ob eine Person den Weg zum Ziel festlegt, priorisieren kann und Aufgaben antizipatorisch kognitiv durchdringt.

Die vormalige Positionsmacht der Führenden wird gleichsam zur Machtzuteilung an das Individuum, das die traditionelle Führungsrolle an sich selbst vollführt. Unter Selbstführung wird zunächst eine individuelle Coping-Strategie verstanden, um effizient und qualitativ hochwertig arbeiten zu können. In einem zweiten Schritt fließen diese individuellen Kompetenzen im Team zusammen: Dort werden gemeinsam Ziele gesetzt, der Arbeitsfortschritt bewertet, Erfolg imaginiert. Selbstführung bedeutet zudem, Spielräume wahrzunehmen und zu nutzen, Zeitspielräume zu sehen, um kreative Ansätze zu finden oder neue Wege zu gehen, um Ziele zu erreichen und 
mit Hindernissen konstruktiv verfahren zu können. Dabei sind sowohl intuitive als auch reflektierte Fähigkeiten und Kenntnisse zum Konzept gehörig.

Da Selbstführungskompetenz sehr stark vom organisatorischen Umfeld abhängt, ist der agile Transformationsraum der, der Selbstführung gleichsam ermöglicht oder gar erzwingt. Dabei kommt der Selbstführung dessen, der die vormalige Macht abgibt, die größte Bedeutung zu:

- balanciert sein,

- frei denken können (jenseits der Managementhoden),

- ein „Charakter“ sein.

Die liebevolle Beziehungsgestaltung zum Team mindert das Einsamkeitserleben, setzt jedoch Ambiguitäts- und Spannungstoleranz voraus. Es reicht eine Führung „good enough“ (Haubl und Möller 2016). Für agile Führung benötigen Führungskräfte mehr denn je nicht nur Sachkompetenz, sondern auch Selbsterfahrung, um die eigenen Wahrnehmungen und Wahrnehmungsverzerrungen von den Wahrnehmungen und Wahrnehmungsverzerrungen ihrer Mitarbeitenden unterscheiden zu können. Es braucht Haltung: Führungskräfte begleiten ihre Mitarbeitenden situationsflexibel - geben ihnen so viel Unterstützung, wie sie in einer konkreten Situation gebraucht wird. Und nicht mehr als das!

- So viel Selbstbestimmung wie möglich!

- So viel Fremdbestimmung wie nötig!

Um den anspruchsvollen Transformationsprozess hin zu mehr Agilität zu gestalten, braucht es vor allem eines: Vertrauen. Vertrauen meint eben nicht Kalkül, sondern die Bereitschaft, sich ins Risiko (auch des Scheiterns) zu begeben: „Wer vertraut, steigert insofern seine eigene Verwundbarkeit, da er sich auf bestimmte Erwartungen hinsichtlich des Verhaltens von Personen oder Systemen verlässt, die nicht vollständig seiner persönlichen Kontrolle unterliegen“ (Gilbert 2006, S. 120).

Luhmann kennzeichnet Vertrauen und Misstrauen als generalisierte oder spezifische Einstellung zu Personen oder Systemen. „Wer Vertrauen erweist, nimmt Zukunft vorweg“, sagt Luhmann (2000, S. 8). Ein wesentliches Kriterium beim Phänomen Vertrauen ist, dass es sich um eine freiwillig kreierte Beziehungsqualität handelt. Vertrauen ist eine formal nicht abgesicherte Vorleistung und gilt oft als Ersatz für formale Verträge. Ein Vertrauender geht ein Risiko ein und macht sich verwundbar, während der Misstrauende dieses Risiko scheut.

Auf der individuellen Ebene spielt also die Bereitschaft der Führungskraft, ihren Mitarbeitenden zu vertrauen, eine Rolle. Wenn es heißt, sich auf das Abenteuer Agilität einzulassen, gilt es ihnen zuzutrauen, dass sie auch ohne stetige Kontrolle ihr Bestes werden geben wollen. Diese Persönlichkeitsdisposition der Vertrauensneigung wird sozialisatorisch erworben (vgl. Schweer 2003) und ist somit Kondensat von Interaktionserfahrung im Privatleben und in den unterschiedlichen beruflichen Stationen. Auf der Führungs- und Teamebene kommt aber vor allem der Vertrauenswürdigkeit eine wichtige Rolle zu. Die Vertrauenswürdigkeit kann, einem empirisch vielfach belegten Modell von Mayer et al. (1995) folgend, auf drei Ebenen eingeschätzt werden, die zu jeweils unterschiedlichen riskanten Vertrauensbeziehungen führen können. Demnach lässt sich 
1. auf die Kompetenzen oder die Fähigkeiten (competece based trust),

2. auf die moralische Integrität (integrity based trust),

3. auf die gute Intention einer anderen Person (goodwill trust) vertrauen.

Mit den drei verschiedenen Zuschreibungen sind unterschiedliche Risiken (Leistungsrisiken, moralische Risiken und gute Intention) verbunden. Als gemeinsames Grundverständnis hat sich in der Vertrauensforschung herauskristallisiert, dass Vertrauen mit einer positiven Erwartungshaltung gegenüber Personen, Gruppen und Institutionen zu tun hat, deren Verhalten durch den Vertrauenden nicht kontrolliert oder sanktioniert werden kann. Agil arbeitende Teams kommen ohne Vertrauensneigung und ohne Vertrauenswürdigkeit nicht aus: Wissen zu teilen und entsprechend der Reziprozitätsnorm: ,,Vertrauen verpflichtet, Vertrauen wird nicht geschenkt“ (vgl. Neuberger 2006, S. 12) zu erwarten, dass andere ebenso handeln. Es gibt einen unausgesprochenen psychologischen Vertrag gleichermaßen geltender Offenheit. Diese ,riskante Haltung“ gibt es auch dem Kund/innensystem gegenüber. Wer vertraut, verzichtet bewusst auf Informationen.

Vertrauen steht vor allem für die Vorhersagbarkeit des Verhaltens des Vertrauensempfängers. Vertrauen steigert die Erwartungssicherheit und kann Zukunftsängste vermindern. Vertrauen ersetzt fehlende Information und sichert trotz Ungewissheit und überschauter Komplexität die Handlungsfähigkeit der Subjekte. Zuversicht, Zuverlässigkeit, Zutrauen und Sich-Verlassenkönnen werden in der Moderne (vgl. Giddens 1995) notwendig, um Kontingenz zu sichern. Wer vertraut, verzichtet auf Überwachung.

\section{Psychologische Sicherheit als Schlüsselkonzept für Selbstorganisation und agile Führung}

Das Konzept „Psychologische Sicherheit“ (Edmondson 1999) steht für die gemeinsame Überzeugung aller Mitglieder eines Teams, dass die Sicherheit innerhalb der Gruppe gegeben ist, zwischenmenschliche Risiken einzugehen. Dazu gehört z.B., mit Lösungsmöglichkeiten zu experimentieren, eigene Fehler einzugestehen und sich von der wahrgenommenen Team-Norm abweichend zu verhalten.

Die Atmosphäre psychologischer Sicherheit zeichnet sich durch folgende Elemente aus:

- Offen die eigene Meinung äußern können. Jedes Mitglied kann ohne Risiko Ideen einbringen und die Entscheidungen anderer infrage stellen; unterschiedliche Meinungen werden respektiert und diskutiert; es herrscht Vertrauen darauf, dass andere nicht versuchen, einen zu untergraben.

- Jede*r spricht gleich viel. Das muss nicht in jedem Meeting gleich verteilt sein, aber sich über einen gewissen Zeitraum ausgleichen.

- Soziale Empathie, die ein gegenseitiges Verständnis erzeugt. Es herrscht eine hohe soziale Sensibilität vor; die Mitglieder des Teams können sich in die Anderen einfühlen und achten auf deren Bedürfnisse. 
- Fehler werden als Lernproblem gesehen. Es gilt, aus Fehlern und Schwächen zu lernen sowie Lösungen anstatt Schuldige zu suchen. Neugier und Experimente werden gefördert.

- Individuelle Stärken, Talente und Fähigkeiten werden geschätzt. In einem Team verfügt jede/r über eigene Talente und Erfahrungen. Der Fokus geht weg von einer defizitären hin zu einer stärkenorientierten Sichtweise.

Große Bekanntheit erlangte das Konzept erst 2016 durch das Forschungsprojekt „Aristoteles“ von Google. Für diese Studie wurden mehr als 180 Teams im Unternehmen untersucht, um die Dynamik von Hochleistungsteams zu beschreiben. Meyer et al. (2018) würdigt die Studie und sieht das wenig theoriegeleitete Vorgehen eher kritisch. Dennoch ist sie wichtiger Ausgangspunkt umfangreicher Forschungsaktivitäten zur psychologischen Sicherheit.

Zahlreiche Arbeiten belegen den Zusammenhang von Führung und psychologischer Sicherheit. Moderne und positive Führungsstile, wie z. B. Shared Leadership und transformationale Führung, wirken sich positiv auf Lernkultur vermittelt durch psychologische Sicherheit aus (Bachmann und Quispe 2021). Im Umkehrschluss bedeutet dies, dass hierarchische und transaktionale Führung ungünstig für die Entwicklung psychologischer Sicherheit ist. Auch die Beziehung zwischen Führungskraft und Teammitgliedern hat einen Einfluss auf die psychologische Sicherheit: Wer in gutem Kontakt zu ihr steht, fühlt sich sicherer und kann mehr Risiken eingehen; wer sich am Rande fühlt, kämpft mit mehr Unsicherheit und bringt sich weniger ein (ebd.).

Vor Edmondson (2008) haben Schein und Bennis (1965) psychologische Sicherheit als Konzept in Zusammenhang von Lewins Theorie der Veränderung von Gruppen und sozialen Systemen (unfreeze - move - freeze) genutzt. Psychologische Sicherheit wird als notwendig gesehen, um die Lernangst bei beginnenden Veränderungen zu überwinden. Metastudien sehen zwei wichtige Tendenzen: Zum einen hängt psychologische Sicherheit stark mit Informationsaustausch und zum anderen mit Lernverhalten zusammen (Meyer et al. 2018). Edmondson (2008) hat psychologische Sicherheit systematisch in Verbindung mit Verantwortungsübernahme gebracht und geht davon aus, dass nur psychologische Sicherheit gepaart mit hoher Verantwortungsübernahme ein Team in die Lernzone bringt (Abb. 4). Wir sehen hier auch einen Zusammenhang zum Führungsstil. Wenn Führungskräfte z.B. durch Prinzipien des Shared Leadership Verantwortungsübernahme fördern, erhöhen sie bei bestehender psychologischer Sicherheit die Lern- und Innovationsfähigkeit ihres Teams.

Wir verwenden psychologische Sicherheit im Führungscoaching und „empowern“ Führungskräfte und Teammitglieder, indem wir ihnen das Konzept zur Selbstreflexion und Selbststeuerung zur Verfügung stellen. Im nachfolgenden Fallbeispiel verwendet die Führungskraft psychologische Sicherheit nicht nur implizit, sondern explizit, indem sie das Konzept im Team einführt und es als Messlatte für die $\mathrm{Zu}$ sammenarbeit verwendet.

Fallbeispiel: Unser Kunde war Leiter eines 12-köpfigen Führungsteams in einem Logistik-Konzern. Er klagte darüber, dass sein Team nicht konstruktiv zusammenarbeiten würde. Er schilderte: Immer wieder wechselten die Teammitglieder 
Abb. 4 Psychologische Sicherheit und Verantwortungsübernahme. (Aus Meyer et al. 2018)

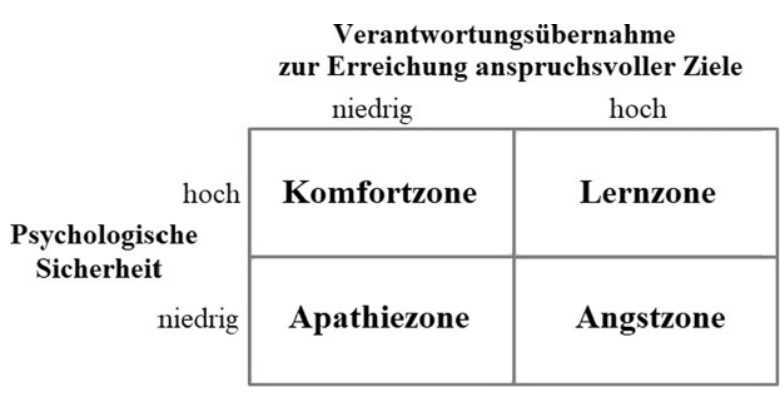

das Thema, kritisierten sich heftig gegenseitig und entwerteten die Vorschläge der Anderen. Sie entwickelten eine Kampf-Flucht-Dynamik (Giernalczyk und Möller 2018). Dann wiederum würde ein besonders erfahrener Kollege das Wort an sich reißen und alle anderen dominieren. Schließlich würde wiederholt über Fehler der jüngsten Vergangenheit in einer Weise gesprochen, bei der die Suche nach dem Schuldigen im Vordergrund stünde. Unser Coachee hatte den Eindruck, dass seine bisherigen Interventionen verpuffen würden. Er war ein sitzungserfahrener Manager und erwog die Möglichkeit, ,dem Spuk“ mit klaren Ansagen und Leitplanken ein Ende zu bereiten. Zurück hielt ihn, dass er mit dieser Leitwolf-Mentalität natürlich die alte hierarchische Welt wieder zurückholen würde und er dem Gedanken agiler Selbststeuerung zuwider handeln würde.

Da er sich nicht auf seine innere Autorität bzw. die Autorität von oben beziehen wollte, schlugen wir ihm vor, mit einer methodischen Autorität zu arbeiten und psychologische Sicherheit als Konzept für seine Leitung in den Mittelpunkt zu stellen.

- Der Coachee führte das Konzept in der folgenden Teamsitzung ein.

- Er moderierte einen Prozess, in dem das Team darüber diskutierte, ob es für sie passend wäre, damit zu arbeiten.

- Anschließend arbeitete er zusammen mit seinem Team an der gemeinsam erstellten inhaltlichen Agenda.

- Während der Sitzung bezog er sich gelegentlich auf das Konzept und erklärte immer wieder sein eigenes Handeln.

- Besonders effektiv erwiesen sich seine Hinweise an das dominante Teammitglied, der unter Bezug zur Dimension ,Jeder spricht gleich viel“ meinte, ,ich glaube, ich sage jetzt mal nichts".

- Außerdem fand das Team einen anderen Zugang zu Fehlern. Sie nahmen sich eine Lessons Learned Phase vor, in der sie Fehler analysieren wollten, um daraus zu lernen.

- Nach einer Phase der inhaltlichen Diskussion führte der Coachee eine Reflexionsphase ein:

- An welchen Stellen haben wir uns an das Konzept (PS) gehalten und wann sind wir abgewichen?

- Darüber hinaus arbeitete er auch mit der Methode der Second Chance: „Wo habe ich etwas nicht gesagt und was hat mich zurückgehalten?“” 
Psychologische Sicherheit steht exemplarisch dafür, wie die Autorität der Führungskraft im agilen Paradigma Schritt für Schritt durch Methoden und Regeln bezüglich der Zusammenarbeit ersetzt wird. Die Führungskraft vermittelt Methodik zur Selbstführung. Auf diese Weise verinnerlichen Teammitglieder die Methoden und entfalten zunehmend mehr Selbstmanagement-Fähigkeiten.

\section{Fazit}

Der notwendige Transformationsprozess in vielen Unternehmen ist ohne die Orientierung an New Leadership nicht zu denken. Um dieser oben skizzierten neuen Rollenanforderung nachzukommen, braucht es eine innere Bewegung der Führungskräfte, die Verzicht auf althergebrachte Sicherheiten bedeutet. Jedoch steht dem „Verlust“ ein massiver Gewinn neuer Rollensegmente der neuen Führungskultur gegenüber. Das Konzept der psychologischen Sicherheit verhilft den Mitarbeitenden und den Führungskräften, sich auf neues und wirksameres Terrain zu wagen.

Funding Open Access funding enabled and organized by Projekt DEAL.

Open Access Dieser Artikel wird unter der Creative Commons Namensnennung 4.0 International Lizenz veröffentlicht, welche die Nutzung, Vervielfältigung, Bearbeitung, Verbreitung und Wiedergabe in jeglichem Medium und Format erlaubt, sofern Sie den/die ursprünglichen Autor(en) und die Quelle ordnungsgemäß nennen, einen Link zur Creative Commons Lizenz beifügen und angeben, ob Änderungen vorgenommen wurden.

Die in diesem Artikel enthaltenen Bilder und sonstiges Drittmaterial unterliegen ebenfalls der genannten Creative Commons Lizenz, sofern sich aus der Abbildungslegende nichts anderes ergibt. Sofern das betreffende Material nicht unter der genannten Creative Commons Lizenz steht und die betreffende Handlung nicht nach gesetzlichen Vorschriften erlaubt ist, ist für die oben aufgeführten Weiterverwendungen des Materials die Einwilligung des jeweiligen Rechteinhabers einzuholen.

Weitere Details zur Lizenz entnehmen Sie bitte der Lizenzinformation auf http://creativecommons.org/ licenses/by/4.0/deed.de.

\section{Literatur}

Bachmann, T., \& Quispe, K. Q. (2021). Wie entsteht psychologische Sicherheit und Teamidentifikation? Eine empirische Untersuchung. OSC, 28(3), 319-337. https://doi.org/10.1007/s11613-021-00717-4. Baecker, D. (1994). Postheroisches Management: Ein Vademecum. Berlin: Merve.

Beck, K., et al. (2001). Manifest für agile Softwareentwicklung. http://agilemanifesto.org/iso/de/manifesto. html. Zugegriffen: 27. Febr. 2021.

Becker, H., \& Langosch, I. (1995). Produktivität und Menschlichkeit. Bd. 4. Stuttgart: Enke.

Bergmann, F. (2017). Neue Arbeit-Neue Kultur (6. Aufl.). Freiburg: Arbor. (2004)

Edmondson, A.C. (1999). Psychological safety and learning behavior in work teams. Administrative Science Quarterly, 44, 350-383.

Edmondson, A.C. (2008). The competitive imperative of learning. HRB, 86(7/8), 60-67.

Giddens, A. (1995). Konsequenzen der Moderne. Frankfurt: Suhrkamp.

Giernalczyk, T., \& Möller, H. (2018). Entwicklungsraum. Psychodynamische Beratung in Organisationen. Göttingen: Vandenhoeck \& Ruprecht.

Gilbert, D. U. (2006). Systemvertrauen in Unternehmensnetzwerken: Eine Positionsbestimmung aus strukturationstheoretischer Perspektive. In K. Götz (Hrsg.), Vertrauen in Organisationen (S. 113-134). München: Rainer Hampp.

Haubl, R., \& Möller, H. (2016). Führung „good enough“. Unveröff. Manuskript. 
Heckhausen, J., \& Heckhausen, H. (2006). Motivation und Handeln. Heidelberg: Springer.

Krug, S., \& Kuhl, U. (2006). Macht, Leistung, Freundschaft. Motive als Erfolgsfaktoren in Wirtschaft, Politik und Spitzensport. Stuttgart: Kohlhammer.

Luhmann, N. (2000). Vertrauen. Ein Mechanismus zur Reduktion sozialer Komplexität. Stuttgart: Enke.

Mayer, R. C., Davis, J.H., \& Schoorman, F. D. (1995). An integrative model of organizational trust. Academy of Management Review, 20(3), 709-734. https://doi.org/10.5465/AMR.1995.9508080335. http:// www.jstor.org/stable/258792?seq=1\&cid=pdf-reference\#fndtn-references_tab_contents [letzter Zugriff am 20. 03. 2017].

McClelland, D. (1975). Power: the inner experience. New York: Halstead.

Meyer, H. A., Wrba, M., \& Bachmann, T. (2018). Psychologische Sicherheit: Das Fundament gelingender Arbeit im Team. In S. Hess \& H. Fischer (Hrsg.), Mensch und Computer 2018 - Usability Professionals. Dresden, 02.-05.Sept. 2018. https://doi.org/10.18420/muc2018-up-0243.

Müller, G.F. (2013). Fragebogen zur Diagnose von Führung durch Selbstführung (FDFSF). Landau: UMBRA-Landau.

Murray, H. A. (1943). Thematic apperception test. Cambridge: Harvard University Press.

Musati, M. (2020). New Work - neue Führung? Rolle von Führungskräften in agilen Unternehmen. Kassel: Universität Kassel. Unveröff. Masterarbeit

Musati, M. (2022). Agilität - ein Führungsmodell für öffentliche Verwaltungen. OSC. https://doi.org/10. 1007/s11613-021-00714-7.

Neck, C.P., \& Manz, C.C. (2013). Mastering self-leadership: empowering yourselffor personal excellence (6. Aufl.). Upper Saddle River: Pearson.

Neuberger, O. (2006). Vertrauen vertrauen? Misstrauen als Sozialkapital. In K. Götz (Hrsg.), Vertrauen in Organisationen (S. 11-55). München: Rainer Hampp.

Rheinberg, F., \& Engeser, S. (2010). Motive training and motivational competence. In O. C. Schultheis \& J. C. Brunstein (Hrsg.), Implicit motives (S. 510-548). New York: Oxford University Press.

Schein, E.H., \& Bennis, W. G. (1965). Personal and organizational change through group methods: The laboratory approach. New York: Wiley.

Schermuly, C. (2019). New Work - Gute Arbeit gestalten: Psychologisches Empowerment von Mitarbeitern (2. Aufl.). Freiburg: Haufe.

Schweer, M. (2003). Vertrauen als Organisationsprinzip: Prinzipien für komplexe soziale Systeme. Bern: Huber.

Steinmann, B., Kleinert, A., \& Maier, G. W. (2020). Promoting the underestimated: a vignette study on the importance of the need for affiliation to successful leadership. Motivation and Emotion. https://doi. org/10.1007/s11031-020-09833-7.

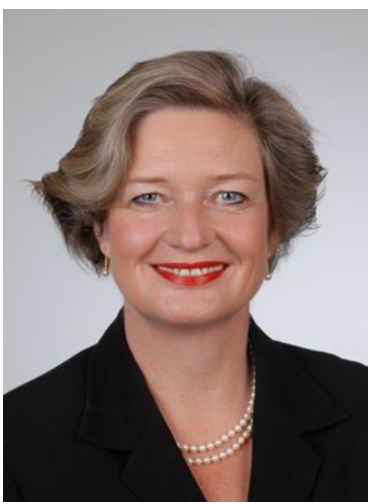

Prof. Dr. Heidi Möller Prof. Dr. phil., Dipl.-Psych., Professorin für Theorie und Methodik der Beratung, Universität Kassel. Psychoanalytikerin, Supervisorin (DGSv) und Lehrsupervisorin, Organisationsberaterin und Coach. Wissenschaftliche Leiterin des postgradualen Masterstudiengangs Coaching, Organisationsberatung, Supervision (COS), UNIKIMS. 


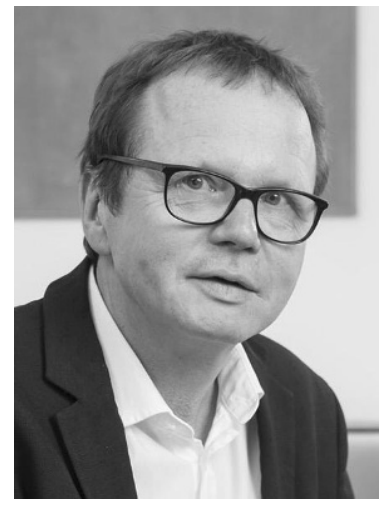

Prof. Dr. Thomas Giernalczyk Prof. Dr. phil., Dipl.-Psych., Psychoanalytiker und Geschäftsführender Gesellschafter von M19-Manufaktur für Organisationsberatung $\mathrm{GmbH}$ (www.m19-organisationsberatung.de), lehrt psychologische und therapeutische Interventionen an der Fakultät für Humanwissenschaften der Universität der Bundeswehr München. Mitgründer des Instituts für Psychodynamische Organisationsberatung München (IPOM), Mitglied der Deutschen Gesellschaft für Supervision und Coaching e. V. (DGSv). www.ipom-net.com. 